\title{
Viscosity modulates blood glucose response to nutrient solutions in dogs
}

\author{
C. Reppas* and J.B. Dressman \\ College of Pharmacy. The University of Michigan, Ann Arbor. MI 48109-1065. USA
}

(Received 4 November 1991)

(Revision accepted 14 April 1992)

\section{Summary}

The relationship between postprandial blood glucose levels and meal viscosity was studied by adding various combinations of hydroxypropylmethylcellulose to glucose solutions and administering them to female mongrel dogs. Glucose was administered as $5 \%$ or $20 \%$ solutions in water. Hydroxypropylmethylcellulose was dissolved in the glucose solutions to yield low $\left(5000 \mathrm{cP}\right.$ measured at $37^{\circ} \mathrm{C}$ and $\left.1 \mathrm{~s}^{-1}\right)$, medium $(15000 \mathrm{cP})$ or high $(30000 \mathrm{cP})$ viscosities. High viscosity hydroxypropylmethylcellulose significantly reduced the maximum blood glucose concentration, $C_{\max }$, by $60 \%(5 \%$ glucose meal $)$ and $40 \%\left(20 \%\right.$ glucose meal) while reducing the area under the blood level vs. time curve $\left(\mathrm{AUC}_{0-3 \mathrm{~h}}\right)$ by $40-50 \%$. Medium viscosity hydroxypropylmethylcellulose reduced the $C_{\max }$ at both glucose levels, but reduced the AUC only for the $5 \%$ glucose meal. Low viscosity HPMC lowered the $C_{\max }$ only after the $5 \%$ glucose meal, and had no significant effect on the AUC at either glucose level. The average time to reach maximum concentration, $T_{\max }$, was prolonged two- to three-fold at all viscosity levels for the $5 \%$ glucose solutions, but was not affected when $20 \%$ glucose solutions were administered. It was concluded that hydroxypropylmethylcellulose can effectively retard the absorption of glucose from the gastrointestinal tract, and that the extent of this effect is related to the viscosity of the solution administered.

Key words: Viscosity; Hydroxypropylmethylcellulose; Water-soluble fiber; Blood glucose; Canine

\section{Introduction}

Fiber appears to be linked with diabetes in that fiber-depleted diets have been associated with the

Correspondence to: J.B. Dressman, College of Pharmacy, The University of Michigan, Ann Arbor, MI 48109-1065, USA.

* Current address: Department of Pharmacy, University of Athens, Panepistimiopolis, 15771 Zografou, Athens, Greece. pathogenesis of diabetes and that increasing the fiber content of the diet has been used successfully to control postprandial glucose levels [1]. The ability of dietary fiber to facilitate glycemic control in diabetes has been studied on both an acute and long term basis by incorporating different types of fiber into test meals and examining postprandial glycemic and hormonal responses. In particular, the water soluble fibers 
such as guar and pectin have been demonstrated to reduce postprandial glucose levels in both type 1 and 2 diabetics, as well as in normal and obese non-diabetic volunteers [2-6]. There is substantial evidence in the literature to show that the ability of a water soluble fiber to form a viscous solution is an important determinant of its ability to affect the postprandial glucose response. For example, in a study by Jenkins et al. [3] it was shown that when guar was hydrolyzed to a non-viscous form, it was no longer able to attenuate the blood glucose response. Furthermore, Wolever et al. [7] found that the effect of guar was greater when the fiber was given in the viscous, hydrated form than in the non-hydrated form.

We have reported previously [8] that hydrodynamic responses in the gut to fiber meals are dependent on the viscosity of the solution administered, using hydroxypropylmethylcellulose (HPMC) as the water soluble fiber. Lumenal viscosity is increased, the lumenal flow rate is slowed and the lag time for gastric emptying is extended when viscous solutions of HPMC are administered. As the input viscosity is raised, the hydrodynamic responses become more pronounced. Based on these observations it was hypothesized that for HPMC, as for guar, meal viscosity is an important determinant of the postprandial blood glucose response.

The aim of the study reported here was to further investigate the relationship between viscosity of a water soluble fiber solution and its ability to affect the postprandial blood glucose response. Specifically, we wished to determine whether there is a cutoff viscosity for fiber effect on blood glucose response, or whether there is a gradual increase in effect with increasing ability of the fiber to elevate the viscosity of the gut contents. We compared the blood glucose response to nonviscous glucose solutions with the response to glucose solutions adjusted to low, medium or high viscosities with various combinations of HPMCs. Use of HPMCs with the same unit structure but differing molecular weights enabled us to obtain a viscosity-response profile over a wide range of viscosities without changing the total fiber concentration or fiber type.

\section{Materials and Methods}

\section{Animals}

Seven female mongrel dogs $(20-28 \mathrm{~kg})$ were used for this study, which was approved by the University committee for the use and care of animals in research.

\section{Preparation of solutions}

Solutions were prepared in the following manner:

(a) Glucose-only solutions, to assess the blood glucose response in the absence of fiber, were prepared at two glucose levels, $5 \%$ and $20 \%$. The $5 \%$ glucose solution consisted of $25 \mathrm{~g}$ glucose [D-( + )-glucose, Sigma Chemical Co., St. Louis, MO], 4 g PEG 4500 (Dow Chemical Co., Midland, MI), and $500 \mathrm{ml}$ distilled water. The $20 \%$ glucose solution consisted of $100 \mathrm{~g}$ glucose, $4 \mathrm{~g}$ PEG 4500 and $500 \mathrm{ml}$ distilled water.

(b) Test solutions consisted of HPMC (Dow Chemical Co., Midland, MI) dissolved in $5 \%$ or $20 \%$ glucose solutions. HPMC was dispersed by heating to $80^{\circ} \mathrm{C}$ in one-third of the glucose solution, then mixing in the rest of the solution and allowing to cool. HPMC solutions were prepared at three viscosity levels: low $(5000 \mathrm{cP}$ at $37^{\circ} \mathrm{C}$ and $\left.1 \mathrm{~s}^{-1}\right)$, medium $(15000 \mathrm{cP})$, and high $(30000 \mathrm{cP})$. The viscous solutions were prepared at a total concentration of $2 \%(10 \mathrm{~g}$ per $500 \mathrm{ml})$ using blends of $\mathrm{K} 4 \mathrm{M}, \mathrm{K} 15 \mathrm{M}$ and $\mathrm{K} 100 \mathrm{M}$ premium grade HPMC (Methocel ${ }^{\circledR}$, Dow Chemical Co., Midland, MI). These grades all consist of the same monomer units, polymerized to successively higher molecular weights. A second medium viscosity HPMC solution (referred to as the K4 solution in the text) was prepared with $3.3 \% \mathrm{~K} 4 \mathrm{M}$ Methocel ${ }^{\circledR}(16.5 \mathrm{~g}$ per $500 \mathrm{ml})$. The combinations of HPMC grades used with the corresponding lot numbers, the percentage of each component required to achieve the desired viscosity level, procedures for the measurement of input viscosity, and techniques for the interpretation of viscosity 
data have been published previously [8]. The mean viscosities of the solutions administered are quoted in Table 1 as the value in centipoise (cP) at $1 \mathrm{~s}^{-1}$ and $100 \mathrm{~s}^{-1}$, measurcd at $37^{\circ} \mathrm{C}$. HPMC is a pseudoplastic material so the viscosity of HPMC solutions varies with the shear rate. Quotation of viscosity characteristics at a single shear rate for such materials may be misleading. Therefore, the consistency index, a constant derived from the overall viscosity/shear profile (range used, $100-1000 \mathrm{~s}^{-1}$ ), is also quoted for each of the solutions as an indication of the 'thickness' of the solution over a wide range of shear rates [8].

(c) Saline solutions, to assess blood glucose response in the absence of glucose, were prepared by incorporating appropriate amounts of sodium chloride $(4.5$ or $17.5 \mathrm{~g}$ per $500 \mathrm{ml})$ to match the osmolalities of the glucose solutions. The $0.9 \%$ $\mathrm{NaCl}$ solution was administered as a nonviscous or as a high viscosity HPMC solution. The $3.5 \%$ solution had to be administered as a high viscosity HPMC solution to avoid emesis.

\section{Study protocol and assay}

Solutions were administered through an orogastric tube after fasting the dog for $16 \mathrm{~h}$ from food but not water. Blood was sampled prior to and $20,40,60,80,100,130,160$ and $180 \mathrm{~min}$ following the administration of the solution. The samples were taken via an indwelling 21-gauge catheter in the foreleg of the dog. Dogs received each solution type on one or, in some cases, two occasions. The maximum frequency of testing according to this protocol was twice per week per dog.

\section{TABLE 1}

Mean $( \pm \mathrm{SD})$ viscosities $\left(\mathrm{cP}\right.$ at $\left.37^{\circ} \mathrm{C}\right)$ and consistency indices (shear rate range: $100-1000 \mathrm{~s}^{-1}$, at $37^{\circ} \mathrm{C}$ ) of the four HPMC meals administered to the dogs

\begin{tabular}{lclc}
\hline Meal & cP at $1 \mathrm{~s}^{-1}$ & $\mathrm{cP}$ at $100 \mathrm{~s}^{-1}$ & Consistency index \\
\hline Low & $6827(3486)$ & $1081(75)$ & $16981(1960)$ \\
Medium & $15122(4798)$ & $1773(105)$ & $44871(3286)$ \\
K4 meal & $16881(6399)$ & $3292(246)$ & $110975(33489)$ \\
High & $29276(5282)$ & $2337(181)$ & $78337(9450)$ \\
\hline
\end{tabular}

Serum glucose levels were measured using a standard kit from Sigma Diagnostics (St. Louis, $\mathrm{MO})$ with a spectrophotometric endpoint.

\section{Data analysis}

For each experiment, the maximum concentration, $C_{\max }$, and the time at which this occurred, $T_{\max }$, could be readily determined from the plot of blood glucose concentration vs. time. In addition, the area under the blood glucose vs, time plot in the $3-h$ postprandial period $\left(\mathrm{AUC}_{0-3} \mathrm{~h}\right.$ ) was calculated using the trapezoidal rule [9].

For each dog, the data for AUC from the experiments with saline solutions were averaged. Likewise, the data for $\mathrm{AUC}$ and $C_{\max }$ from the glucose-only solutions were averaged. Then, for each experiment in which a HPMC/glucose combination was administered, the $C_{\max }$ and AUC were determined. To obtain the $C_{\max }$ in the test solution experiment as a percentage of the $C_{\max }$ in the glucose-only solution for each experiment, the average concentration of blood glucose observed after administration of saline solutions to that dog was first subtracted. For example, for a dog with an average blood glucose level of $4 \mathrm{mM}$ after administration of saline solutions, and an average $C_{\max }$ of $9 \mathrm{mM}$ after administration of glucose-only solutions, the $C_{\text {max }}$ following the test meal could be expressed as a percentage of the glucose-only meal value, $\% C_{\max }^{*}$, using the following formula:

$$
\%_{0} C_{\max }^{*}=\left\{\left[C_{\max }(\text { test })-4\right] /[9-4]\right\} \times 100
$$

Use of each dog as its own control was necessary because of the large interdog variation in glucose levels. A similar procedure was used to calculate $\% \mathrm{AUC}^{*}$ values. By contrast, the $T_{\max }$ in the test solution as a percentage of the $T_{\max }$ in the glucose-only solution for each experiment, $\% T_{\max }$, was obtained by direct comparison of the two values.

The $\% C_{\text {miax }}^{*}, \% \mathrm{AUC}^{*}$ and $\% T_{\max }$ values from individual experiments were then averaged to obtain the mean values in each dog. Mean values for 
each dog were then averaged to obtain the Grand Mean values.

Statistical analysis was performed by using the Statworks ${ }^{\circledR}$ statistical package for Macintosh (Data Metrics, Inc., Philadelphia, PA), with $n$ reflecting the number of dogs used for each experimental condition, and differences were tested for significance at the 0.05 level. Differences between the glucose-only solutions and the specific test solution with respect to each of the pharmacokinetic parameters were assessed by Student's $t$-test for paired data. Linear regression analysis was applied to correlate the viscosity of the prepared meals with pharmacokinetic parameters.

\section{Results}

Saline solutions. The Grand Mean fasting glucose level was $3.85 \pm 0.94 \mathrm{mmol} / 1(\bar{x} \pm \mathrm{SD})$. This value is in good agreement with values reported in the literature for dogs [10] and close to the usual range for humans (4-6.5 mmol/1 according to the University of Michigan Hospital guidelines). Administration of normal saline as an osmotic control for the $5 \%$ glucose meal had no effect on the blood glucose levels. Nor were the blood glucose levels affected by the addition of HPMC when the HPMC was incorporated in either a $0.9 \%$ or $3.5 \%$ saline solution (sec Fig. 1). Cocfficients of variation from the fasting level for the saline meals during the first $3 \mathrm{~h}$ after administration ranged from 6 to $17 \%$ among the seven dogs.

Glucose-only solutions. After administration of $5 \%$ glucose solutions, the maximum glucose concentration ranged from 6.3 to $13.3 \mathrm{mmol} / 1$, average $9.4 \mathrm{mmol} / \mathrm{l}$. There was also considerable variability in the maximum blood glucose level reached after administering a $20 \%$ glucose meal, where the maximum blood concentration ranged from 6.7 to $17.1 \mathrm{mmol} / 1$, average $11.7 \mathrm{mmol} / 1$. The time to maximum concentration, $T_{\max }$, ranged widely from 20 to $80 \mathrm{~min}$ for the $5 \%$ meals and from 40 to $160 \mathrm{~min}$ for the $20 \%$ meals. In many cases, the $C_{\max }$ and $T_{\text {max }}$ values observed fell within the range of values usually encountered in
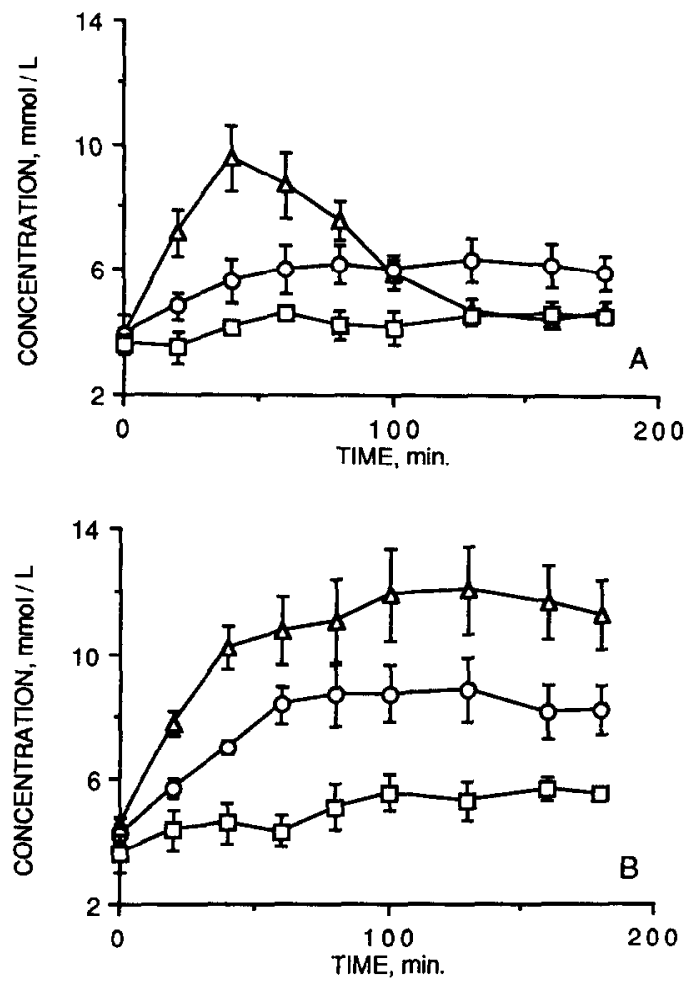

Fig. 1. Postprandial blood glucose concentration following administration of $500 \mathrm{ml}$ meals containing $5 \%$ (A) or $20 \%$ (B) solutions of glucose. Triangles indicate glucose-only meals; squares indicate saline (A) or $3.5 \% \mathrm{NaCl}$ plus high viscosity HPMC (B) meals; circles represent results from test meals (glucose plus high viscosity HPMC). Each point represents the Grand Mean \pm SEM from five dogs.

diabetic human subjects. Grand Mean blood glucose vs. time curves for the glucose-only meals are shown in Fig. 1 using data pooled from all dogs studied.

Test solutions. Figure 1 illustrates the Grand Mean blood glucose levels observed after administration of the test solutions. Grand Mean values for $\% C_{\max }^{*}, \% \mathrm{AUC}^{*}$ (calculated according to Equation 1) and $\% T_{\max }$ are listed in Table 2 for the $5 \%$ glucose solutions and in Table 3 for the $20 \%$ glucose solutions.

Coadministration of HPMC with a $5 \%$ glucose solution resulted in an average decrease in the adjusted $C_{\max }$ to around $40-60 \%$ of the adjusted value for the $C_{\text {max }}$ in the glucose-only experiments. As viscosity increases, $\% C_{\max }^{*}$ decreases, but in 
TABLE 2

Mean pharmacokinetic parameters for $5 \%$ glucose meals containing various levels of HPMC expressed as $\% C_{\max }^{*}$, $\%_{0}$ AUC $^{*}$ and $\% T_{\max }{ }^{a}$

\begin{tabular}{|c|c|c|c|c|}
\hline Meal & $\% C_{\max }^{*}$ & $\% T_{\max }$ & $\mathrm{AUC}_{0-3 \mathrm{~h}}^{*}$ & $n^{\mathrm{b}}$ \\
\hline Low & $55(35)^{*}$ & $262(161)$ & $92(42)$ & $4 / 5$ \\
\hline Medium & $56(19)^{*}$ & $233(153)$ & $63(39)$ & $3 / 4$ \\
\hline K4 meal & $56(34)$ & $236(164)$ & $82(46)$ & $3 / 4$ \\
\hline High & $38(12)^{*}$ & $340(174)^{*}$ & $46(20)^{*}$ & $5 / 6$ \\
\hline
\end{tabular}

a $\mathrm{SD}$ is given in parentheses. Asterisks denote statistically significant differences from the positive control meals at the 0.05 level

b Number of dogs studied/total number of experiments run. Repeated measures were made on selected dogs for each viscosity level studied.

TABLE 3

Mean pharmacokinetic parameters for $20 \%$ glucose meals containing various levels of HPMC expressed as $\% \mathrm{C}_{\max }^{*}$, ${ }_{0}^{\circ} \mathrm{AUC}^{*}$ and ${ }_{0} T_{\max }{ }^{\mathrm{a}}$

\begin{tabular}{llrll}
\hline Meal & $\%_{\text {o }}^{*} C_{\max }$ & $\%_{\mathrm{o}} T_{\text {lllax }}$ & $\%$ AUC $_{0-3 \mathrm{~h}}^{*}$ & $n^{\mathrm{b}}$ \\
\hline Low & $84(41)$ & $102(20)$ & $74(36)$ & $4 / 6$ \\
Medium & $52(11)^{*}$ & $58(18)$ & $47(12)^{*}$ & $3 / 4$ \\
K4 meal & $61(23)^{*}$ & $78(46)$ & $57(20)$ & $3 / 4$ \\
High & $63(14)^{*}$ & $92(25)$ & $57(16)^{*}$ & $5 / 10$
\end{tabular}

a $\mathrm{SD}$ are given in parentheses. Asterisks denote statistically significant differences from the positive control values at the 0.05 level.

b Number of dogs studied/total number of experiments run. Repeated measures were made on selected dogs for each viscosity level studied.

a nonlinear fashion. Low and medium viscosity test solutions produced a similar decrease in $\% C_{\max }^{*}$, while the highest viscosity HPMC resulted in a greater reduction. In the case of the $20 \%$ glucose solutions, the low viscosity HPMC produced only a trend to reduce the $C_{\text {max }}$, while the reduction was significant for the two medium and the high viscosity HPMC solutions. The relationship between $\% \mathrm{C}_{\max }^{*}$ and solution viscosity was not adequately described by a linear equation for the $20 \%$ glucose meals since the reduction in $C_{\text {max }}$ appeared to plateau for the medium and high viscosity test solutions.
Addition of HPMC to the $5 \%$ glucose solutions tended to reduce the area under the curve $\left(\mathrm{AUC}_{0-3 \mathrm{~h}}\right)$. The reduction was dramatic in the case of the high viscosity polymer, while for the medium and low viscosity HPMC solutions the difference from positive control values did not reach significance at the 0.05 level. Linear regression between solution viscosity (measured at $37^{\circ} \mathrm{C}$ and at $1 \mathrm{~s}^{-1}$ ) and $\% \mathrm{AUC}_{0-3 \mathrm{~h}}^{*}$ indicated that a significant inverse correlation exists between these two parameters. This relationship is shown in Fig. 2A. For solutions containing 20\% glucose, the $\mathrm{AUC}_{0-3 \mathrm{~h}}$ values also tended to be reduced when HPMC were added, and the effect was as much as a $40-50 \%$ reduction when medium or high viscosity HPMC combinations were used. The medium and high viscosity HPMC so-
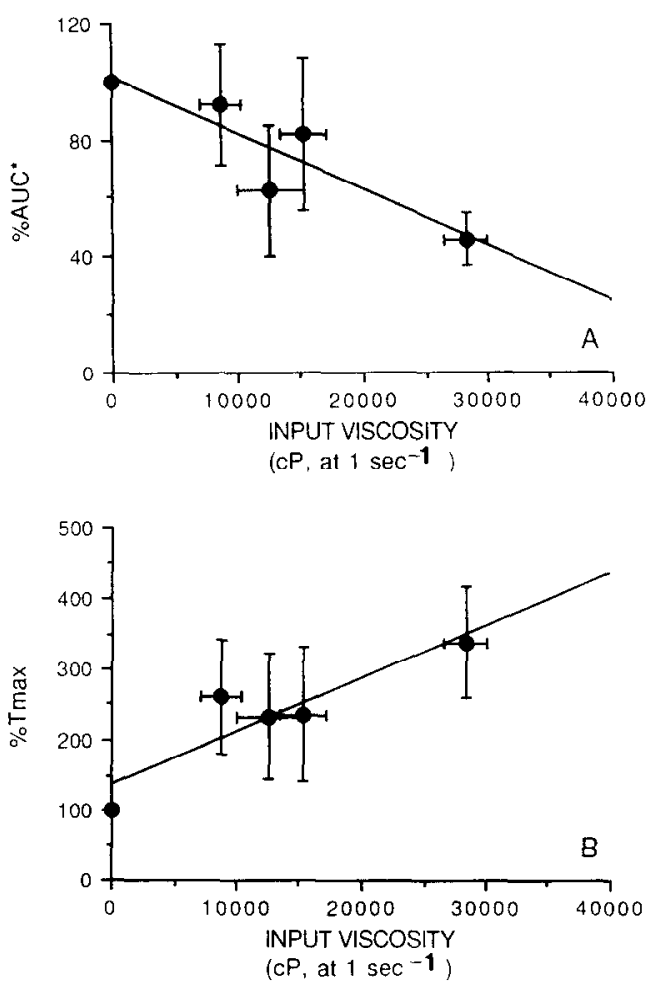

Fig. 2. Correlation of meal viscosity (mean \pm SEM) with (A) percent maximum rise in AUC, ${ }_{0}^{\circ}$ AUC $^{*}(r=-0.900$, $P=0.04$ ), and (B) percent maximum change in time at which the maximum blood level occurred, ${ }_{0} T_{\max }(r=0.909$, $P=0.03$ ), for the $5 \%$ glucose meals. 
lutions reduced $\mathrm{AUC}_{0-3 \mathrm{~h}}$ to a significant degree, whereas the low viscosity and $\mathrm{K} 4$ solution did not.

The addition of HPMC to the $5 \%$ glucose solutions resulted in an average increase in the $T_{\max }$ by a factor of two to three, but this was statistically significant only with the high viscosity test solution. Figure 2B shows the relation between viscosity (measured at $37^{\circ} \mathrm{C}$ and at $1 \mathrm{~s}^{-1}$ ) and $T_{\max }$ for the $5 \%$ glucose solutions. $T_{\max }$ was consistently longer after the $20 \%$ than after the $5 \%$ glucose-only solutions, but the addition of HPMC did not result in any further increase.

\section{Discussion}

The results indicate that the high viscosity HPMC used in this study was effective in controlling both the maximum serum level and the area under the serum level versus time profile (a measure of the extent of glucose absorption) when glucose solutions were administered to dogs. Regression analysis indicated that there were significant linear correlations between the mean percentage change of the $\mathrm{AUC}_{0-3 \mathrm{~h}}(r=-0.900, P=0.04)$ and $T_{\text {max }}{ }^{-}$ parameters $(r=0.909, P=0.03)$ and the input viscosity when $5 \%$ glucose solutions (Fig. 2) were administered. Although the relationship was not linear, there was also a clear trend for high viscosity HPMC solutions to reduce the $C_{\max }$ more than low and medium viscosity solutions, which in turn produced lower $C_{\max }$ values than the glucose-only solution. These relationships suggest that, in the case of $5 \%$ glucose solutions, the input viscosity of the solution is a key factor in the ability of the water soluble fiber to control the blood glucose profile.

The reduction in $C_{\max }$ and $\mathrm{AUC}_{0-3 \mathrm{~h}}$ was less dramatic when HPMCs were added to the $20 \%$ glucose solutions. Although the medium and high viscosity HPMC solutions were about equally effective in controlling the serum glucose profile, the low viscosity HPMC solution had no significant effect. As with the $5 \%$ glucose solutions, these results indicate that the blood glucose re- sponse is a graded effect of viscosity, rather than an all-or-none effect. The $T_{\max }$ values after administration of $20 \%$ glucose solutions were not affected significantly by the addition of HPMC. Presumably, negative feedback effects on gastric emptying are already maximal when a $20 \%$ glucose solution is administered so that the addition of HPMC does not result in any additional effect.

To determine the relative importance of molecular weight and concentration effects, the results for the two medium viscosity HPMC solutions were compared. Addition of the $2 \%$ high molecular weight HPMC to the glucose solutions resulted in more effective control of serum glucose than the $3.3 \%$ low molecular weight (K4M) HPMC. Using a higher molecular weight fiber rather than increasing the concentration of a lower molecular weight grade thus appears to be the more efficient way of controlling the serum glucose profile.

Further studies necd to be done to clarify the mechanisms by which HPMC modifies the postprandial blood glucose response. However, it appears that viscosity and molecular weight of the fiber are two important factors. Preliminary experiments in three mongrel dogs fistulated at midgut showed a tendency for the amount of glucose recovered from the fistula to increase when the high viscosity fiber was added to the glucose solutions. Recovery increased from an average of $20 \%$ to an average of $30 \%$ of the glucose load administered. These data suggest that the mechanism involves a decrease in the diffusivity of glucose in the lumen thereby confirming that lumenal events are important to the mechanism of this fiber effect on glucose absorption. Nevertheless, several other properties may potentially affect efficiency of a water soluble fiber to modify postprandial blood glucose response. An important consideration for fibers ingested in solid form is the rate at which they hydrate in the gut. This effect has already been demonstrated for guar by Wolever et al. [7]. Another factor related to viscosity is the shear-thinning properties of the fiber (viscosity under high motility conditions vs. low motility conditions). In addition, the fiber's abil- 
ity to bind water, ions, bile salts and other components of the GI fluids may influence the blood glucose response. From these considerations, it is not expected that all water soluble fibers will be equally effective in controlling blood glucose. Jenkins et al. [3], for example, compared five types of fiber and an ion-exchange resin and found that of these, only guar, methylcellulose and tragacanth significantly reduced blood glucose levels.

Further studies also need to be conducted to determine whether the effects observed with HPMC coadministered with glucose solutions also apply to the ingestion of ordinary meals. Although 5 and $20 \%$ glucose solutions in a volume of $500 \mathrm{ml}$ induced fed state physiological responses in the GI tract, there are important differences to solid meals. For example, the behavior of nutrient solutions is quite different to that of ordinary solid meals [11]. In addition, ordinary meals will contain complex as well as simple carbohydrates. Both of these factors will alter the rate at which glucose is presented to and taken up by the absorbing mucosa.

Of the water soluble fibers studied in the treatment of diabetes, guar is the most widely used, followed by pectin. Although guar is one of the most viscous naturally occurring polysaccharides and is effective in reducing postprandial blood glucose [3], its lack of palatability [12] and tendency to produce flatulence owing to partial digestion in the colon restricts its widespread use. Further, batch to batch variation in rheological behavior and the inherently low hydration rate may lead to variable efficacy [3,13]. Attempts to overcome these problems by incorporating the guar in bread [14,15], formulating in a slowgelling preparation [16-18] or preparation of a frozen sherbet formulation in the case of pectin [19] have not met with complete success [18,20].

The identification of other viscous polysaccharides that are more palatable and at least equally effective [21] may be a useful strategy for improving fiber supplementation as an approach to the treatment of diabetes. In studies when guar was given with a hypertonic glucose solution [3,5,2123] the minimum concentration of guar effective in lowering blood glucose levels (for chosen time intervals) was $3 \%$, with doses ranging from 9 to $14.5 \mathrm{~g}$ per meal. Three studies have compared area under the blood level vs. time profiles of glucose with and without guar $[3,7,21]$. These studies found that guar significantly reduced the AUCs when these were measured during the first one or two hours after the meal, but did not report significance over longer intervals. These observations are consistent with claims that guar delays but does not reduce carbohydrate absorption $[13,24]$. The large reductions in both $C_{\max }$ and AUC observed in the current study with $2 \%$ solutions of high molecular weight HPMC suggest that this fiber may warrant further investigation as an agent for improving glucose tolerance.

\section{Acknowledgements}

We express our sincere thanks to the Dow Chemical $\mathrm{Co}$. for financial support of this investigation and to Mr John Wlodyga for his excellent technical assistance.

\section{References}

1 Pilch, S.M. (Ed.) (1987) Physiological Effects and Health Consequences of Dietary Fiber. Life Sciences Research Office. FASEB, pp. 79-92.

2 Jenkins, D.J.A., Goff, D.V., Leeds, A.R. et al. (1976) Unabsorbable carbohydrates and diabetes: decreased postprandial hyperglycemia. Lancet ii, 172-174

3 Jenkins, D.J.A., Wolever, T.M.S., Leeds, A.R. et al. (1978) Dietary fiber, fiber analogues and glucose tolerance: importance of viscosity. Br. Med. J. 1, 1392-1394.

4 Krotkiewski, M. (1984) Effect of guar gum on body weight, hunger ratings and metabolism in obese subjects. Br. J. Nutr. 52, 97-105.

5 Blackburn, N.A., Holgate, A.M. and Read, N.W. (1984) Does guar gum improve postprandial hyperglycemia in humans by reducing small intestinal contact area? Br. J. Nutr. 52, 197-204.

6 Morgan, L.M., Goulder, T.J., Tsiolakis, D. et al. (1979) The effect of unabsorbable carbohydrate on gut hormones. Diabelologia 17, 85-89.

7 Wolever, T.M.S., Jenkins, D.J.A., Nineham, R. et al. (1979) Guar gum and reduction of postprandial glycemia: 
effect of incorporation into solid food, liquid food, and both. Br. J. Nutr. 41, 505-510.

8 Reppas, C., Meyer, J., Sirois, P. et al. (1991) Effect of hydroxypropylmethylcellulose on GI transit and lumenal viscosity in dogs. Gastroenterology 100, 1217-1223.

9 M. Rowland and T.N. Tozer (Eds.), (1980) Clinical Pharmacokinetics. Concepts and Applications. Lea and Febiger, Philadelphia, pp. 288-289.

10 J.R. Duncan and K.W. Prasse (Eds.) (1986) Veterinary Laboratory Medicine. Iowa State University Press, Ames, Iowa, Chapter 6.

11 Meyer, J.H. (1987) Motility of the stomach and gastroduodenal junction. In: L.R. Johnson (Ed.) Physiology of the Gastrointestinal Tract. Raven Press, New York, Chapter 19.

12 Jenkins, D.J.A., Taylor, R.H., Nineham, R. et al. (1979) Combined use of guar and acarbose in reduction of postprandial glycaemia. Lancet ii, 924-927.

13 Seaman, J.K. (1980) In: R.L. Davidson (Ed.), Handbook of Water-soluble Gums and Resins. McGraw-Hill Book Co., New York, Chapter 6.

14 Jenkins, D.J.A., Wolever, T.M.S., Nineham, R. et al. (1978) Guar crispbread in the diabetic diet. Br. Med. J. 2, 1744-1746.

15 Ellis, P.R., Apling, E.C., Leeds, A.R. et al. (1981) Guar bread: acceptability and efficacy combined. Studies on blood glucose, serum insulin and satiety in normal subjects. Br J. Nutr. 46, 267-276.

16 Aro, A., Uusitupa, M., Voutilainen, E. et al. (1981) Im- proved diabetic control and hypocholesterolaemic effect induced by long-term dietary supplementation with guar gum in type 2 (insulin-independent) diabetes. Diabetologia 21, 29-33.

17 Ray, T.K., Mansell, K.M., Knight, L.C. et al. (1983) Long term effects of dietary fiber on glucose tolerance and gastric emptying in noninsulin-dependent diabetic patients. Am. J. Clin. Nutr. 37, 376-381.

18 O'Conner, N., Tredger, J. and Morgan, L. (1981) Viscosity differences between various guar gums. Diabetologia 20, 612-615.

19 Monnier, L., Pham, T.C., Aguirre, L. et al. (1978) Influence of indigestible fibers on glucose tolerance. Diabetes Care 1, 83-88.

20 Cohen, M. and Martin, F.I.R. (1979) Guar crispbread in the diabetic diet. Br. Med. J. 1, 616-617.

21 Edwards, C.A., Blackburn, N.A., Craigen, L. et al. (1987) Viscosity of food gums determined in vitro related to their hypoglycemic actions. Am. J. Clin. Nutr. 46, 72-77.

22 Lembcke, B., Ebert, R., Caspary, W.F. et al. (1984) Role of gastrointestinal transit in the delay of absorption by viscous fibre (guar). Hepatogastroenterology 31, 183186.

23 Blackburn, N.A., Redfern, J.S., Jarjis, H. et al. (1984) The mechanism of action of guar gum in improving glucose tolerance in man. Clin. Sci. 66, 329-336.

24 Jenkins, D.J.A., Wolever, T.M.S. and Bacon, S. (1980) Diabetic diets: high carbohydrate combined with high fiber. Am. J. Clin. Nutr. 33, 1729-1733. 